THU0077 INCREASED FOLLICULAR HELPER T CELL REGULATES AUTOANTIBODY HYPOSIALYLATION IN GLUCOSE-6-PHOSPHATE ISOMERASE INDUCED ARTHRITIS

I. Kurata, I. Matsumoto, A. Osada, H. Ebe, H. Kawaguchi, Y. Kondo, H. Tsuboi, T. Sumida. Internal Medicine, University of Tsukuba, Tsukuba, Ibaraki, Japan

Background: Circulating follicular helper $\mathrm{T}$ (Tfh) cells were reported to be increased and promote B cell activation and antibody production in rheumatoid arthritis. Recently, IL-23-Th17 cells axis and hyposialylation of antibodies were proved to be linked to the inflammation of experimental and rheumatoid arthritis. However it remains uncertain how Tfh, including IL-17 producing Tfh (Tfh17), is associated to arthritis and whether its function includes promotion of antibody hyposialylation.

Objectives: The aim of this study is to explore the relation between Tfh and autoantibody hyposialylation in glucose-6-phosphate isomerase (GPI) induced arthritis (GIA), which mouse model was dependent on T cells, B cells and IL-17. Methods:

1. Fluctuation of Tfh and its subsets in draining lymph nodes (dLNs) were analysed and expression of co-stimulatory molecules were assessed among these subsets. Their localization were examined by immunofluorescence (IF) staining. In order to explore the counterpart reaction, serial changes of plasmablast and plasma cell population in dLNs were also analysed.

2. To elucidate Tfh function in ex-vivo, naïve B cells were co-cultured with Tfh and the ratio of differentiated plasmablast was quantified. Anti-GPI antibody production from plasmablast was measured in the existence of Tfh

3. The titers of anti-GPI antibodies in GIA sera were measured by ELISA.

4. DCs were stimulated with purified anti-GPI antibodies from day 7 (arthritis onset phase) and day 28 (resolving phase) GIA to examine the pathogenicity change of antibody. MRNA of ST6 beta-galactoside alpha2,6-sialyltransferase 1 (st6gal1), the responsible protein for antibody sialylation, in plasmablast was quantified by PCR and detection of sialic acid in anti-GPI antibody was performed by lectin blotting.

5. Naïve B cells were co-cultured with Tfh and the st6gal1 expression in differentiated plasmablast was measured by flow cytometry.

Results:

1. Tfh cells were increased in GIA. It peaked at day 7, the onset of arthritis, and Tfh17 was specifically increased at the same time. Moreover, OX40 expression in Tfh17 was higher than other subsets. IF showed that Tfh and Tfh17 were accumulated in germinal centre of dLNs. As counterparts, plasmablasts and plasma cells were most increased at day 7 as well.

2. When co-cultured with Tfh, the frequency of differentiated plasmablast was much higher than other conditions, and anti-GPI antibody production was up-regulated in the existence of Tfh and GPI.

3. Conflicting with the results above, anti-GPI antibody titers in the sera were gradually elevated even after day 7 and this elevation continued while GIA peaked out.

4. DCs produced higher level of TNF-alpha when stimulated with the antibody from day 7 GIA than day 28. St6gal1 expression in plasmablast was significantly decreased at day 7 and recovered at day 28. In addition, the day 7 antibodies were tended to be contain less sialic acid.

5. Decreased expression of st6gal1 was observed in differentiated plasmablast co-cultured with Tfh.

Conclusions: Tfh, especially Tfh17 were increased in the induction phase of arthritis. Also, Tfh could have a crucial role in the development of arthritis via plasmablast activation and regulation of autoantibody hyposialylation in GIA

Disclosure of Interest: None declared

DOI: 10.1136/annrheumdis-2018-eular.2865

\section{THU0078 BERBERINE AMELIORATES BONE EROSIONS IN COLLAGEN-INDUCED ARTHRITIS RAT MODELS VIA SUPPRESSING THE EXPRESSION OF IL-17A}

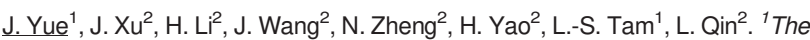
department of Rheumatology; ${ }^{2}$ Department of Orthopedics and Traumatology, Prince of Wales Hospital, The Chinese University of Hongkong, Hongkong, Hong Kong

Background: Rheumatoid arthritis (RA) is a chronic progressive disease characterised by synovial inflammation, autoantibody production, cartilage and bone destruction. Bone erosions are a key feature of RA reflecting both disease severity and disease progression. An imbalance between Th17 and regulatory T cells (Treg cells) has been extensively recognised in both patients and model animals of RA. Oral administration of berberine, an isoquinoline alkaloid, has been showed to ameliorate various symptoms of autoimmune diseases including RA.

Objectives: To verify whether berberine may prevent bone erosions during RA progression and to explore the potential mechanisms in Collagen-induced arthritis (CIA) rat model.

Methods: The severity of arthritis was expressed as mean arthritic index on a 0-4 scale according to the following criteria: $0=$ no oedema or swelling; $1=$ slight oedema and erythema limited to the foot and/or ankle; $2=$ slight oedema and erythema from the ankle to the tarsal bone; $3=$ moderate oedema and erythema from the ankle to the tarsal bone; and $4=$ severe oedema and erythema from the ankle to the entire leg. Each limb was graded, and thus the maximum possible score was 16 for each animal. The threshold score of rats with established CIA is 2. The CIA rats were divided into 3 groups: placebo group $(n=4)$, low dose berberine group (50 mg/kg/day, $\mathrm{n}=4)$ and high dose berberine group $(200 \mathrm{mg} / \mathrm{kg} /$ day $\mathrm{n}=4)$. Placebo and berberine were intragastrically administered to all rats for 4 and 8 weeks after the CIA models were established. TNF- $\alpha$, IL-1 $\beta$, IL-6, IL-17A, and $\lg$ in the serum were measured by ELISA kits (purchased from Abcam). The hind paws of rats were scanned by micro CT (Scanco, Switzerland).

Results: The thickness of the swollen hind paws was reduced in the high dose berberine group ( $200 \mathrm{mg} / \mathrm{kg} /$ day) compared with the placebo group (Fig A). No significant differences were observed in the levels of TNF- $\alpha$, IL-1 $\beta$, and IL-6 between the three groups. However, the levels of IL-17A and IgG were significantly decreased in the high dose berberine group when compared with the placebo group (Fig A). Micro CT data revealed that berberine could significantly improve the microstructure of $\mathrm{CIA}$ rats including the bone volume ratio (BV/TV), areal bone mineral density (aBMD) and trabecular separation (Tb Sp) (Fig B and C). Development of bone erosion had also been partially prevented.
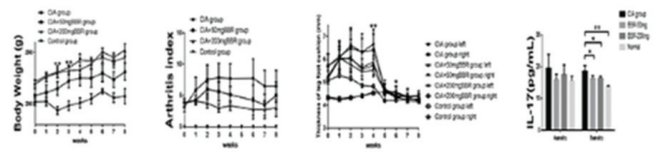

Mean $\pm S D, A N O V A .{ }^{*} P<0.05 ;{ }^{* *} P<0.01$
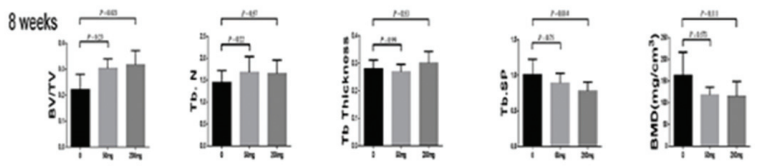

Mean $\pm S D$, ANOVA
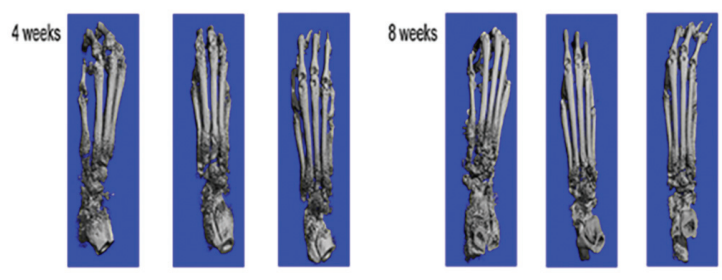

0

$50 \mathrm{mg} / \mathrm{kg} / \mathrm{day} 200 \mathrm{mg} / \mathrm{kg} / \mathrm{day}$

0

$50 \mathrm{mg} / \mathrm{kg} / \mathrm{day} \quad 200 \mathrm{mg} / \mathrm{kg} / \mathrm{day}$

Figure $1 \mathrm{~A}$; B microstructure; C 3D reconstruction

Conclusions: Berberine attenuated the symptoms of CIA rats and may prevent bone erosion progression by suppressing IL-17A in CIA. Human studies are required to confirm whether it may serve as a potential treatment for RA in the future.

Disclosure of Interest: None declared

DOI: 10.1136/annrheumdis-2018-eular.1991

\section{THU0079 \\ ADIPONECTIN AGGRAVATES BONE EROSION BY PROMOTING OSTEOPONTIN PRODUCTION IN SYNOVIAL TISSUE OF RHEUMATOID ARTHRITIS}

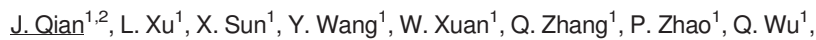
R. Liu ${ }^{1}$, N. Che ${ }^{1}$, F. Wang ${ }^{3}$, W. Tan ${ }^{1}$, M. Zhang ${ }^{1}$. 'Department of Rheumatology and Immunology, The First Affiliated Hospital of Nanjing Medical University, Nanjing; ${ }^{2}$ Department of Rheumatology and Immunology, Affiliated Hospital of Nantong University, Nantong; ${ }^{3}$ Department of Cardiology, The First Affiliated Hospital of Nanjing Medical University, Nanjing, China

Background: We have previously reported that adiponectin (AD), an adipokine that is secreted by adipocytes, correlates closely with progressive bone erosion in rheumatoid arthritis (RA). The exact mechanism of $A D$ towards promoting joint destruction remain unclear. 
Objectives: Osteopontin (OPN) is required for osteoclast recruitment. We hypothesised that $A D$ exacerbates bone erosion by inducing OPN expression in synovial tissue. This study aimed to evaluate a novel role for AD in RA.

Methods: The serum levels of AD and OPN were determined in 38 RA, 40 osteoarthritis $(O A)$ patients, and 20 healthy controls using enzyme-linked immunosorbent assay (ELISA). $A D$ and OPN production were measured by double immunofluorescence of RA and OA synovial tissue. Quantitative real-time PCR and immunofluorescence were used to evaluate the mRNA and protein expression levels of OPN in RA synovial fibroblasts (RASFs) and OA synovial fibroblasts after preincubation with AD, respectively. Migration of the RAW264.7 osteoclast precursors cell line was assessed using the Transwell migration assay and co-culture system. Bone destruction and osteoclastogenesis were assessed by immunohistochemistry, microcomputed tomography, and tartrate-resistant acid phosphatase (TRAP) staining in AD-treated collagen-induced arthritis ( $\mathrm{CIA}$ ) mice with or without OPN silencing. The expression levels of OPN and integrin $\alpha_{v} \beta_{3}$ in the ankle joint tissues of the mice were examined by double immunofluorescence. Results: Our results indicated that the AD and OPN expression levels increased noticeably and were associated with each other in the RA serum. The AD distribution was coincident with that of OPN in the RA synovial tissue. AD stimulation of RASFs increased OPN production in a dose-dependent manner. AD-treated RASFs promoted RAW264.7 cell migration, and the effect was blocked with a specific antibody against OPN. Silencing of OPN using lentiviral-OPN short hairpin RNA reduced the number of TRAP-positive osteoclasts and the extent of bone erosion in the AD-treated CIA mice. When bound to integrin $\alpha_{v} \beta_{3}$, OPN functions as a mediator of $A D$ and osteoclasts.

Conclusions: Our study provides new evidence of AD involvement in bone erosion. $A D$ induces the expression of OPN, which recruits osteoclasts and initiates bone erosion. These data highlight AD as a novel target for RA treatment.

Disclosure of Interest: None declared

DOI: 10.1136/annrheumdis-2018-eular.3379

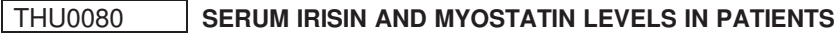 WITH RHEUMATOID ARTHRITIS}

J.M.D.S. Silva ${ }^{1,2}$, R. C. D. E. Santo ${ }^{1,2}$, E.C. Freitas ${ }^{1,2}$, N.F.T. Braz ${ }^{3}$, É. L. M. Vieira $^{3}$, M. F. B. R. Guimarães ${ }^{4}$, A. C. S. E. Silva ${ }^{3}$, A.M. Kakehasi ${ }^{5}, \underline{R}$. M. Xavier $^{2,6}{ }^{1}$ Laboratório de Doenças Autoimunes, Hospital de Clínicas de Porto Alegre; ${ }^{2}$ Universidade Federal do Rio Grande do Sul, Porto Alegre; ${ }^{3}$ Laboratório Interdisciplinar de Investigação Médica, Universidade Federal de Minas Gerais; ${ }^{4}$ Serviço de Reumatologia, Hospital das Clínicas da Universidade Federal de Minas Gerais; ${ }^{5}$ Universidade Federal de Minas Gerais, Belo Horizonte; ${ }^{6}$ Serviço de Reumatologia, Hospital de Clínicas de Porto Alegre, Porto Alegre, Brazil

Background: Rheumatoid arthritis (RA) patients have loss of muscle mass. The balance between muscle protein synthesis and degradation is regulated by cytokines and growth factors, named myokines, such as irisin and myostatin. Myokines are mainly expressed by skeletal muscle and exert systemic effects promoting crosstalk among different tissues. Irisin increases cortical bone mass and its low levels are related to muscle atrophy and obesity[, ${ }^{1,2}$ while myostatin is a negative regulator of muscle growth and regeneration and has a direct role in osteoclastogenesis of inflammatory bone destruction[. . $^{3,4}$

Objectives: To evaluate serum levels of irisin and myostatin and body composition of RA patients and controls.

Methods: 122 female patients with RA, mean age 53 years, mean disease activity score (DAS28) 4.09, mean disease duration 11.2 years and mean body mass index $27.33 \mathrm{~kg} / \mathrm{m}^{2}$ were included. 69 age and sex-matched healthy subjects were enrolled as control group. Irisin (Phoenix Pharmaceuticals) and myostatin ( $R$ and D Systems) serum levels were evaluated by ELISA. Fat mass index (FMl;Kg/m²) and appendicular lean mass index $\left(\mathrm{ALMl} ; \mathrm{Kg} / \mathrm{m}^{2}\right.$ ) were assessed by total body dual-energy $\mathrm{x}$-ray absorptiometry. Student's t test and Spearman correlation were performed. Significance was set at $\mathrm{p}<0.05$.

Table 1. Irisin and myostatin serum levels of RA patients and controls

\begin{tabular}{lccc}
\hline \multicolumn{4}{l}{ Table 1. Irisin and myostatin serum levels of RA patients and controls } \\
\hline & $n$ & $\begin{array}{c}\text { Irisin (mean } \\
\pm S D)\end{array}$ & $\begin{array}{c}\text { Myostatin (mean } \\
\pm S D \text { ) }\end{array}$ \\
\hline RA patients treated with biologics & 13 & $31,71 \pm 7,69^{\#}$ & $2448,64 \pm 1114,90^{* \#}$ \\
RA patients non-treated with & 27 & $25,93 \pm 6,89$ & $3261,66 \pm 1156,28^{+}$ \\
biologics & & & \\
Controls & 30 & $30,36 \pm 10,95$ & $4049,08 \pm 1610,01$ \\
\hline
\end{tabular}

${ }^{*} \mathrm{p}<0,05$ RA patients treated with biologics vs controls; ${ }^{*} \mathrm{p}<0,05$ RA patients treated with biologics vs RA patients non-treated with biologics; $+p<0,05$ RA patients non-treated with biologics vs controls.

Results: RA patients had decreased serum levels of irisin $(25,61 \pm 8,25$ vs 30,36 $\pm 10,95 \mathrm{ng} / \mathrm{ml} ; \mathrm{p}<0.05)$ and myostatin $(3011,28 \pm 1271,11$ vs $4049,08 \pm 1610,01 \mathrm{pg} /$ $\mathrm{ml} ; \mathrm{p}<0.05)$, decreased ALMI $(6,09 \pm 0,88$ vs $6,50 \pm 1,10 ; \mathrm{p}<0.05)$ and increased FMI $(11,26 \pm 3,30$ vs $9,44 \pm 2,65 ; \mathrm{p}<0.05)$, compared to controls. No correlations were observed among irisin and myostatin levels and ALMI and FMI. Of the 122 RA patients, 40 were analysed for the use of biologic medication. Serum levels of irisin and myostatin were different between RA patients treated and non-treated with biologics (table 1).

Conclusions: RA patients presented loss of lean mass and gain of fat mass, as well as lower irisin and myostatin serum levels, in comparison with controls. Additionally, the use of biologic medication by patients impacted on myokines serum levels. Further analyses are needed for a better comprehension of irisin and myostatin roles in RA, and to verify their correlation to other RA features.

\section{REFERENCES:}

[1] Colaianni G, et al. Proc Natl Acad Sci 2015;112(39):12157-62.

[2] Chang J, et al. Geriatr Gerontol Int. 2017;17(11):2266-2273.

[3] Huang Z, et al. Cell Signal 2011;23(9):1441-6.

[4] Dankbar B, et al. Nat Med 2015;21(9):1085-90.

Disclosure of Interest: None declared

DOI: 10.1136/annrheumdis-2018-eular.7214

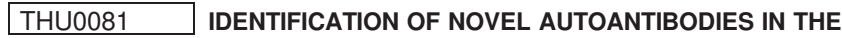 SYNOVIAL FLUID FROM PATIENTS WITH RHEUMATOID ARTHRITIS}

K.-I. Goto ${ }^{1}$, T. Kawamoto ${ }^{2}$, A. Nakajima ${ }^{3}$, M. Tahara ${ }^{4}$, T. Ebata ${ }^{1} .{ }^{1}$ Orthpaedic Surgery, Sakura Orthpaedic Hospital, Sakura; ${ }^{2}$ Orthpaedic Surgery, Matsudo City Hospital, Matsudo; ${ }^{3}$ Orthpaedic Surgery, Toho Univ. Sakura Hospital, Sakura; ${ }^{4}$ Orthpaedic Surgery, Chiba-East National Hospital, Chiba, Japan

Background: Rheumatoid arthritis (RA) is a chromic, autoimmune and inflammatory joint disease with a poorly understood etiology. Despite widespread diagnos tic use of anti-citrullinated protein antibodies and rheumatoid factor, there is strong demand for novel biomarkers to improve the diagnosis this disease.

Objectives: The purpose of present study is to investigate novel autoantibodies in the synovial fluid of RA patients.

Methods: 1) By using SEREX (Serological identification of antigens by recombinant cDNA expression cloning), we identified ten and several antigens from sera of RA patients. 2) Three epitope sites in the candidate antigens proteins were predicted and 18 mer peptides were synthesised. 3) Synovial fluid of the knees was obtained from 48 RA and 48 osteoarthritis (OA) patients. 4) Furthermore, AlphaLISA was used to analyse the antibody levels in synovial fluid using synthetic polypeptide as antigens

Results: Significantly higher proportion of antibodies against lamin A (LMNA, RA $19871+13924$ VS OA $6726+3975, p<0.0000001)$ and cell growth-regulating nucleolar protein (CGRN, RA 19673+13314 VS OA 10614+6391, p<0.00007) were found in synovial fluid of RA as compared with OA.

Conclusions: We identified two novel autoantibodies in the knee synovial fluid of RA patients. These antibodies would have the potential to become diagnostic biomarkers of RA.

\section{REFERENCES}

[1] Taimen $\mathrm{P}$, Pfleghaar $\mathrm{K}$, Shimi $\mathrm{T}$, et al. A progeria mutation reveals functions for lamin A in nuclear assembly, architecture, and chromosome organization. Proc Natl Acad Sci U S 2009;106:20788-93.

[2] Su L, Hershberger RJ, and Weissman IL. LYAR, a nobel nucleolar protein with zinc finger DNA-binding mitifs, is involved in cell growth regulation. Genes Dev 1993;7:735-48.

Disclosure of Interest: None declared

DOI: 10.1136/annrheumdis-2018-eular.3257

\section{THU0082 IMPAIRED LEFT VENTRICULAR RELAXATION AND ITS ASSOCIATION WITH INFLAMMATORY MARKERS IN COLLAGEN-INDUCED ARTHRITIS}

L. Mokotedi ${ }^{1}$, F.S. Michel ${ }^{1}$, C. Mogane ${ }^{1}$, P.H. Dessein ${ }^{2}$, A.M. Millen ${ }^{1} .{ }^{1}$ Physiology, University of the Witwatersrand, Parktown, Johannesburg, South Africa;

${ }^{2}$ Rheumatology Division, Vrije Universiteit Brussel, Brussels, Belgium

Background: Patients with rheumatoid arthritis (RA) experience an increased risk of developing heart failure with a preserved ejection fraction. Although there is some evidence to support a role of chronic inflammation in the pathogenesis of impaired left ventricular (LV) function in RA, ${ }^{1}$ the direct effects of inflammatory cytokines on the LV function in collagen-induced arthritis (CIA) (an experimental model most similar to $\mathrm{RA}$ ) require further elucidation. 